\title{
Depletion of SENP1 suppresses the proliferation and invasion of triple-negative breast cancer cells
}

\author{
ZHONGHUA WANG ${ }^{1,2^{*}}$, JIA JIN $^{1,2^{*}}$, JIAN ZHANG ${ }^{1,2}$, LEIPING WANG ${ }^{1,2}$ and JUN CAO $^{1,2}$ \\ ${ }^{1}$ Department of Medical Oncology, Fudan University Shanghai Cancer Center; \\ ${ }^{2}$ Department of Oncology, Shanghai Medical College, Fudan University, Shanghai 200032, P.R. China
}

Received March 24, 2016; Accepted August 5, 2016

DOI: $10.3892 / o r .2016 .5036$

\begin{abstract}
To investigate the roles of SUMO-specific protease 1 (SENP1) in triple-negative breast cancer (TNBC), we detected the expression level of SENP1 in the clinical samples of TNBC and non-TNBC patients by using immunohistochemical staining, qRT-PCR, and western blotting. We found that SENP1 was highly expressed in the TNBC tissues compared with the normal breast tissues, as well as non-TNBC tissues. Next, we constructed the si-SENP1-transfected TNBC cell lines for further biological function investigation. Then a xenograft model of nude mice was constructed to confirm the roles of SENP1 in TNBC formation and metastasis. According to our results, deficiency of SENP1 significantly represses the proliferation, invasion, migration, and colony formation of TNBC cells through blocking the cell cycle and regulating the expression of cyclin-dependent kinases (CDKs) p21 and p27, as well as MMP9. Furthermore, in vivo experiments revealed that depletion of SENP1 remarkably inhibits the tumor volume, lung metastatis and the expression of p21, p27, MMP9, and Ki67. Taken together, this study elucidated that SENP1 is essential for TNBC cell proliferation and migration in vitro, as well as tumor formation and metastasis in vivo, indicating that SENP1 is a potential therapeutic target for TNBC treatment.
\end{abstract}

\section{Introduction}

Despite the rapid development of diagnosis and treatment in recent decades, breast cancer is still the second leading cause of cancer death in women (1). Based on clinical, pathological, and genetic findings, it is comprised of distinct subtypes (2),

Correspondence to: Professor Zhonghua Wang, Department of Medical Oncology, Fudan University Shanghai Cancer Center, 270 Dong An Road, Shanghai 200032, P.R. China

E-mail: zhonghuawang95@hotmail.com

\section{*Contributed equally}

Key words: triple-negative breast cancer, SUMO-specific protease 1, proliferation, invasion, therapeutic target one of which is triple-negative breast cancer (TNBC). TNBCs are distinguished by lack of estrogen receptor (ER), progesterone receptor (PR), and amplification of the human epidermal growth factor receptor 2 (HER2) (3), accounts for $>15 \%$ of all breast cancers (4) and are more prevalent in young, African-American, and Latino women. Compared with other breast cancer subtypes, TNBC patients have more aggressive clinical course, higher rate of distant recurrence and poorer prognosis $(3,5,6)$. Thus, it is essential to develop novel diagnostic and therapeutic methods for TNBC patients.

Sentrin/SUMO-specific protease 1 (SENP1), a member of SUMO-specific protease (SENP) family, is widely distributed in the nuclei $(7,8)$. SENP1 can deconjugate large numbers of sumoylated proteins. Previously, many studies reported that SENP1 was overexpressed in human prostate cancer (CaP) cells (9), as well as lung cancer tissues (10) and most of colon cancer tissues (11). The expression of SENP1 was reported to have strong prognostic impact on a molecularly defined subset of CaPs (12). Another study revealed that SENP1 might be a prognostic marker and a therapeutic target for metastasis in $\mathrm{CaP}$ patients, for its roles in regulating MMP2 and MMP9 through the HIF-1 $\alpha$ signaling pathway (13). Additionally, SENP1 3'-untranslated region was demonstrated as a regulative target of miR-145, which plays an important role in tumorigenesis of $\mathrm{CaP}$ (14). It was reported that the miR-138/SENP1 cascade was relative to radiosensitization in lung cancer cells (15). Silencing of SENP1 expression results in upregulation of cyclin-dependent kinase (CDK) inhibitors such as p16, p19, p21 and p27, further inhibits cell growth and colony formation in colon cancer cell line, suggesting that SENP1 is essential for cell growth in the colon cancer cell line (11). Moreover, clinical data showed that SENP1 was positively associated with lymph node metastasis and TNM stage of pancreatic cancer (16). In Burkitt's lymphoma, downregulation of SENP1 expression can reduce the stability of HIF-1 $\alpha$ protein and induce the apoptosis of Daudi cells (17). Since the important roles of SENP1 in various cancers, the expression level of SENP1 and whether it is involved in TNBC still requires investigation.

In this study, we firstly investigated the expression of SENP1 in the clinical samples of TNBC and non-TNBC patients by using qRT-PCR, western blotting, and immunohistochemical staining. Our results indicated that SENP1 was highly expressed in TNBC tissues compared with normal 
breast tissues, as well as non-TNBC tissues. To further investigate the roles of SENP1 in TNBC, we constructed si-SENP1-transfected TNBC cell lines, followed by biological function detection. Furthermore, we explored the mechanisms behind the effects of SENP1 in TNBC cells. Additionally, to explore the effects of SENP1 on TNBC tumorigenesis and development, si-SENP1-transfected TNBC cells were transplanted into nude mice to establish TNBC mouse xenograft model. Our research demonstrated the high expression of SENP1 in TNBC cell lines and the negative effect of si-SENP1 on proliferation, invasion, migration, and colony formation ability of TNBC cell lines via blocking of cell cycle and regulation of p21, p27, and MMP9, as well as the suppression effect of SENP1 deficiency on tumor volume, proliferation, and lung metastasis in TNBC tumor-bearing mice. The results of this study revealed the inhibition effect of SENP1 deficiency on tumor proliferation and migration both in vitro and in vivo, indicating the potential roles of SENP1 to promote tumorigenesis and metastasis in TNBC.

\section{Materials and methods}

Ethics statement. The protocols employed in this study and the use of human tissues were approved by the Ethics Committee of Fudan University Shanghai Cancer Center and conducted in full accordance with ethical principles, including the World Medical Association Declaration of Helsinki, and the local legislation. All of the experiments were undertaken with the understanding and written consent of each subject according to the above mentioned principles.

Tissue specimens and immunohistochemistry. Two independent cohorts totaling 82 cases of breast cancer patients were obtained from 2014 to 2015 at Fudan University Shanghai Cancer Center (non-TNBC, $n=42$; TNBC, $n=40$ ). The matched adjacent tissues were obtained from the tissues that were located $5 \mathrm{~cm}$ away from the tumor margin. Immunohistochemical staining was performed according to the manufacturer's instructions. Paraffin-embedded TNBC and adjacent tissues were deparaffinized in $60^{\circ} \mathrm{C}$, followed by treatment with xylene and graded alcohol. The slides were blocked with $5 \%$ bovine serum albumin for $1 \mathrm{~h}$, and then incubated with rabbit anti-human SENP1 polyclonal antibody (cat. no. ab58417) or rabbit anti-human Ki67 polyclonal antibody (both from Abcam, Cambridge, MA, USA; cat. no. ab66155), then visualized by the standard avidin-biotin-peroxidase complex method. Hematoxylin was used for counterstaining. Morphologic images were observed using Olympus fluorescence microscopy (Olympus Corp., Tokyo, Japan).

Cell culture. Normal breast cell lines (HMLE and MCF10A), non-TNBC cell lines (MCF7, T47D, and ZR-75-1), and TNBC cell lines (MDA-MB-231, MDA-MB-453, and MDA-MB-468) were purchased from ATCC (Rockville, MD, USA) and all cell lines except HMLE were routinely maintained in RPMI-1640 medium (Gibco, Carlsbad, CA, USA) supplemented with $10 \%$ fetal bovine serum (FBS) (Invitrogen, Carlsbad, CA, USA) and $50 \mathrm{U} / \mathrm{ml}$ penicillin - $50 \mu \mathrm{g} / \mathrm{ml}$ streptomycin antibiotics (Gibco). HMLE cells were cultured in DMEM/F12 containing growth factor, insulin, hydrocortisone, $100 \mathrm{U} / \mathrm{ml}$ penicillin, and $100 \mu \mathrm{g} / \mathrm{ml}$ streptomycin. Cell lines were cultured in a $37^{\circ} \mathrm{C}$ incubator with humidified atmosphere of $5 \% \mathrm{CO}_{2}$.

Cell transfection. The retrovirus containing SENP1 siRNA or non-specific siRNA as described previously (8) was transfected into TNBC cells to generate si-SENP1-transfected TNBC cells (si-SENP1) or non-specific siRNA-transfected cells (si-NS) via puromycin selection.

Real-time PCR. Total RNA was isolated by TRIzol reagent (Invitrogen). The reverse transcription reactions were performed by cDNA Synthesis Kit (Takara Biotechnology Co., Ltd., Dalian, China) following the manufacturer's protocol. RT-PCR was performed using a standard SYBR PrimeScript RT-PCR Kit (Takara Biotechnology Co., Ltd.) on ABI 7700 real-time PCR system (Applied Biosystems, Foster City, CA, USA). Each sample was analyzed in triplicate and standard deviations representing experimental errors were calculated. SENP1 forward, 5'ATCAGGCAGTGAAACGTTGGAC3' and reverse, 5'GCAGGCTTCATTGTTTATCCCA3'; p21 forward, 5'ACCTCTCAGGGCCGAAAAC3' and reverse, 5'TAGGGC TTCCTCTTGGAGAA3'; p27 forward, 5'CAGAGGACACAC ACTTGGTAGA3' and reverse, 5'TCTTTTGTTTTGAGGA GAGGAA3'; MMP9 forward, 5'AGTCCACCCTTGTGCTC TTCC3' and reverse, 5'TGCCACCCGAGTGTAACCAT3'; $\beta$-actin forward, 5'CTTTTCCAGCCTTCCTTCTTGG3' and reverse, 5'CAGCACTGTGTTGGCATAGAGG3'.

Western blotting. Cells were washed with PBS and lysed. Protein concentration was measured by BCA assay with BCA Protein Assay Kit (Takara Biotechnology Co., Ltd.; cat. no. T9300). The protein extracts were equally loaded onto $10 \%$ SDS polyacrylamide gels, electrophoresed and transferred to PVDF membranes. The membranes were blocked in $5 \%$ fat-free milk in TBST buffer for $2 \mathrm{~h}$ at room temperature. The rabbit anti-human polyclonal antibody was used in conjunction with $0.4 \mu \mathrm{g} / \mathrm{ml}$ of anti-species-conjugated horseradish peroxidase. Rabbit anti-human SENP1 polyclonal antibody (cat. no. ab58417), rabbit anti-human p21 polyclonal antibody (cat. no. ab7960), rabbit anti-human p27 polyclonal antibody (cat. no. ab7961), rabbit anti-human MMP9 polyclonal antibody (cat. no. ab38898) and rabbit anti-human $\beta$-actin polyclonal antibody (cat. no. ab8227) were purchased from Abcam. The bands were detected by chemiluminescent substrate kit (Millipore Corp.). The intensities of the bands were quantified using the NIH ImageJ software package.

Cell growth assay. Cells were seeded into 6-well plates at a density of $1 \times 10^{5}$ cells/well and cultured in the growth medium. Cell growth was measured by MTT assay and recorded by a Benchmark Microplate Reader (Bio-Rad Laboratories, Inc.) at $0,2,4$ and 6 days.

Soft agar colony formation assay. Agarose (0.7\%) was mixed with cells and placed above the $1.2 \%$ agarose underlay. After 2 weeks, colonies were dyed with crystal violet $(0.01 \%$ solution). Then washed with PBS, the colonies were imaged using a custom, automated plate imager with a digital camera (Olympus SP-350; Olympus Corp.). 
Cell cycle assay. Cells were collected and fixed using $75 \%$ ice-cold ethanol at $-20^{\circ} \mathrm{C}$ overnight, then cells were treated with Tris- $\mathrm{HCl}$ buffer ( $\mathrm{pH}$ 7.4) contained with $100 \mu \mathrm{g} / \mathrm{ml}$ RNase A and dyed with propidium iodide (PI; $25 \mu \mathrm{g} / \mathrm{ml}$ ). Then, cell cycle distribution was monitored via flow cytometry. The data were collected and analyzed using ModFit software.

Transwell assay. Cells in logarithmic growth phase were treated with trypsin, washed with PBS and then resuspended in serum-free medium. A total of $4 \times 10^{6}$ cells were added to the upper compartment of the invasion chamber, and $500 \mu 1$ of culture medium were added to the lower compartment of the invasion chamber. Each insert was precoated with $45 \mu \mathrm{g}$ Matrigel (BD Biosciences, San Jose, CA, USA). The Matrigel invasion chambers were incubated at $37^{\circ} \mathrm{C}$ for $48 \mathrm{~h}$ in $5 \% \mathrm{CO}_{2}$. The non-invading cells were removed from the upper face of the membrane and the invaded cells on the lower surface were stained with $0.05 \%$ crystal violet for $30 \mathrm{~min}$. After washed with PBS, the invaded cells were subjected to microscopic inspection (DFC500; Leica Microsystems GmbH, Wetzlar, Germany) according to the manufacturer's instructions. Finally, the values for invasion were obtained by counting three fields per membrane. Experiments were independently repeated in triplicate.

Wound healing assay. Cells were seeded into the bottom of 6-well plates marked a horizontal line on the back for targeting the same field of vision. A line wound was made by scraping a 100- $\mu$ l tip across the confluent cell layer. Cells were washed three times to remove detached cells and debris. Cells were incubated with serum-free medium in a humidified atmosphere containing $5 \% \mathrm{CO}_{2}$ at $37^{\circ} \mathrm{C}$. After cultured for $24 \mathrm{~h}$, wound closure was performed under a light microscope (DFC500; Leica Microsystems $\mathrm{GmbH}$ ) and measured using AxioVision version 4.7 software (Carl Zeiss Meditec, Dublin, CA, USA). Experiments were independently repeated in triplicate.

Xenograft tumor generation. A 50- $\mu 1$ suspension containing $5 \times 10^{6}$ tumor cells (si-SENP1 or si-NS cells) was injected subcutaneously into one side of hind flanks of 6-week-old female BALB/c nude mice. The mice were divided into two groups: si-SENP1 $(n=8)$ and si-NS $(n=8)$ group. After 7 days, when tumors had reached a measurable size, tumor size was determined with a caliper and calculated weekly on weeks 1-8. The experiment was repeated three times. After 10 weeks, the mice were sacrificed, and the number of nodules per lung was recorded.

Statistical analysis. The data are expressed as means \pm SD and analyzed using GraphPad Prism 5.0 software (GraphPad Software Inc.). Data comparison between the two groups was carried out using the two-tailed Student's t-test. For clinical data, p-value was analyzed by Chi-square test using SPSS 17.0. $\mathrm{P}<0.05$ was considered statistically significant.

\section{Results}

SENP1 was highly expressed in TNBC. Clinical data from TNBC and non-TNBC patients were collected and compared. Statistical analysis of clinical data indicated that the TNBC
Table I. Relationship between tumor characteristics and non-triple-negative/triple-negative status.

\begin{tabular}{|c|c|c|c|}
\hline Characteristics & $\begin{array}{c}\text { Non-triple-negative } \\
\mathrm{n}(\%)\end{array}$ & $\begin{array}{c}\text { Triple-negative } \\
\mathrm{n}(\%)\end{array}$ & P-value \\
\hline \multicolumn{4}{|c|}{$\begin{array}{l}\text { Age at } \\
\text { diagnosis (years) }\end{array}$} \\
\hline$\leq 50$ & $10(24)$ & $16(40)$ & 0.015 \\
\hline$>50$ & $32(76)$ & $24(60)$ & \\
\hline \multicolumn{4}{|l|}{ Tumor grade } \\
\hline 1 & $2(5)$ & $0(0)$ & $<0.001$ \\
\hline 2 & $19(45)$ & $8(20)$ & \\
\hline 3 & $21(50)$ & $32(80)$ & \\
\hline \multicolumn{4}{|l|}{ Histological type } \\
\hline Ductal & $29(69)$ & $34(85)$ & $<0.001$ \\
\hline Lobular & $13(31)$ & $6(15)$ & \\
\hline \multicolumn{4}{|l|}{ Tumor size $(\mathrm{cm})$} \\
\hline$\leq 2$ & $21(50)$ & $22(55)$ & NS \\
\hline$>2$ & $21(50)$ & $18(45)$ & \\
\hline \multicolumn{4}{|l|}{ LN status } \\
\hline Negative & $24(57)$ & $19(47)$ & NS \\
\hline Positive & $18(43)$ & $21(53)$ & \\
\hline \multicolumn{4}{|l|}{ LVI status } \\
\hline Negative & $32(76)$ & $26(65)$ & NS \\
\hline Positive & $10(24)$ & $14(35)$ & \\
\hline
\end{tabular}

Data were analyzed by Chi-square test using SPSS 17.0. LN, lymph node; LVI, lymphovascular invasion; NS, non-significant.

was significantly correlated with decreased age $(\mathrm{P}<0.05)$, increased tumor differentiation grade $(\mathrm{P}<0.001)$, and ductal histological type $(\mathrm{P}<0.001)$ (Table $\mathrm{I})$. To investigate the expression levels of SENP1 in TNBC patients, the tumor tissues and adjacent normal breast tissues were collected from TNBC and non-TNBC patients. Through immunohistochemical staining, qRT-PCR and western blotting, we found that SENP1 was highly expressed in breast cancer tissues compared with the adjacent normal tissues. Additionally, a markedly higher level of SENP1 was observed in TNBC tissues, compared to non-TNBC tissue (Fig. 1A-C). We then evaluated the level of SENP1 mRNA and protein expression in normal mammary gland cell lines (HMLE and MCF10A), breast cancer cell lines (MCF7, T47D, and ZR-75-1), as well as TNBC cell lines (MDA-MB-231, MDA-MB-453, and MDA-MB-468). Consistent with the tumor tissue results, both SENP1 mRNA and protein were expressed significantly higher in MDA-MB-231 and MDA-MB-453 than other cell lines (Fig. 1D and E). These results suggested the potential carcinogenic roles of SENP1 in TNBC.

Depletion of SENPI inhibited the proliferation and colony formation of TNBC cells. Due to the high level of SENP1 in TNBC tumor tissues, we subsequently investigated the function of SENP1 in TNBC cell proliferation. Two TNBC cell lines, MDA-MB-231 and MDA-MB-453, were transfected 
A
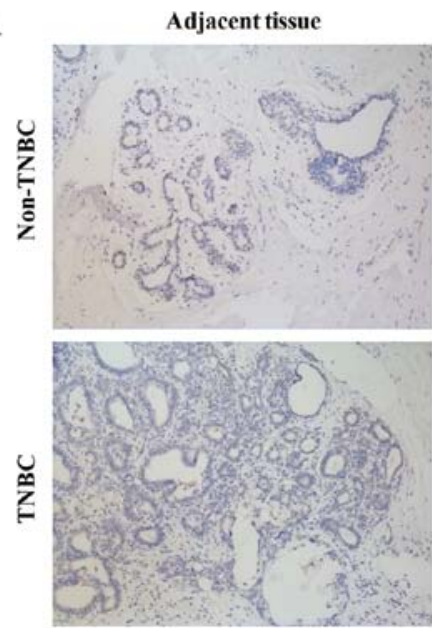

C
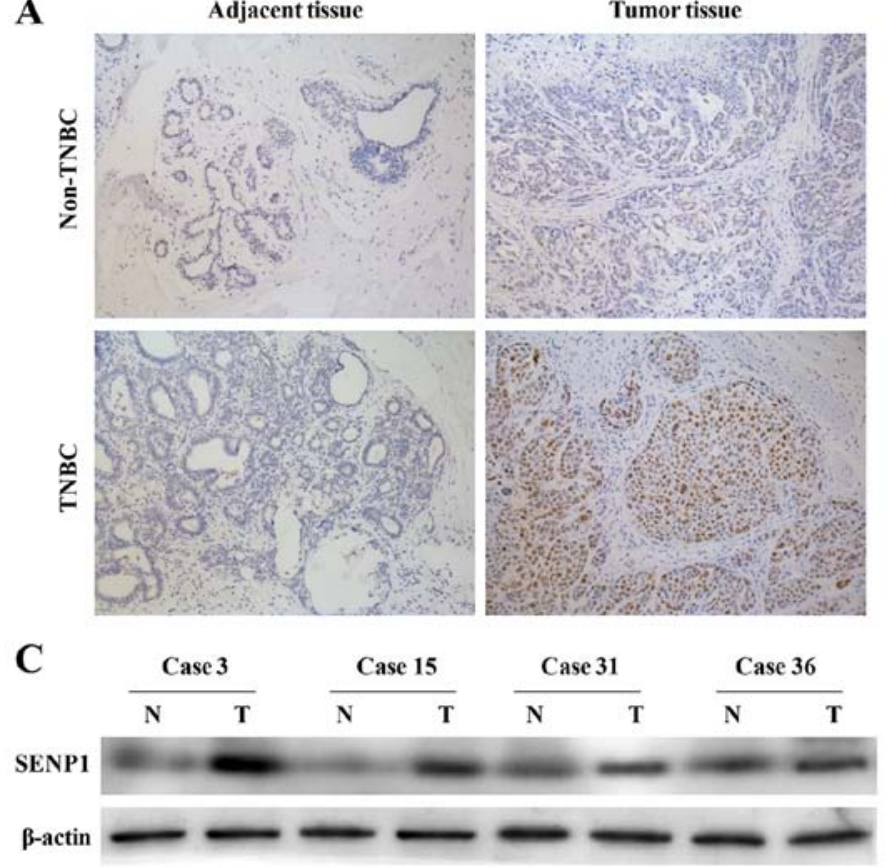

B
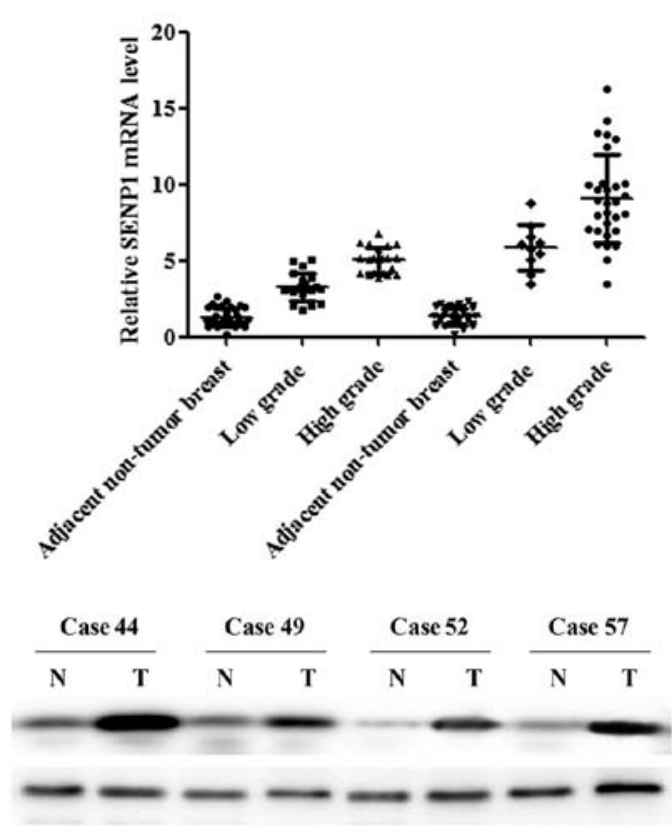

D

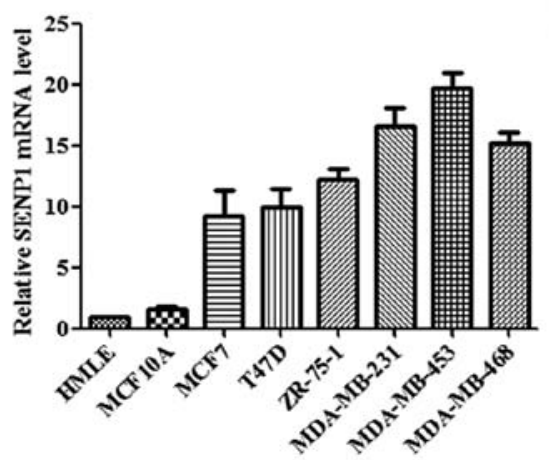

E

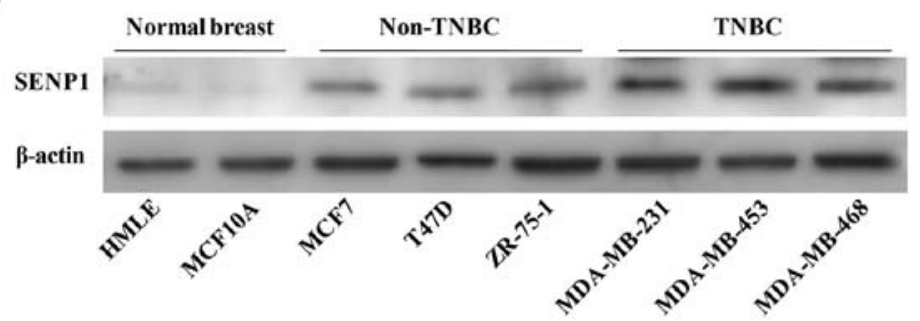

Figure 1. SENP1 is upregulated in TNBC tissues. (A) IHC analysis (magnification, x200), (B) qRT-PCR analysis and (C) western blotting of SENP1 expression in different breast cancers and corresponding adjacent tissue samples. (D) qRT-PCR assay of SENP1 mRNA level and (E) western blotting of SENP1 protein expression in normal breast tissue and breast cancer cell lines. SENP1, SUMO-specific protease 1; TNBC, triple-negative breast cancer.

with si-SENP1 and non-specific RNA, as si-NS, respectively. The transfection efficiency was evaluated via qRT-PCR and western blotting (Fig. 2A and B). The mRNA levels of SENP1 in si-SENP1-infected cells were $<40 \%$ compared with si-NS-infected cells. Through MTT assay, we found that knockdown of SENP1 significantly inhibits the proliferation of TNBC cells (Fig. 2C and E) (5.194 \pm 0.162 vs. 3.283 \pm 0.278 in MDA-MB-231 and 4.546 \pm 0.482 vs. 3.048 \pm 0.207 in MDA-MB-453, respectively). Consistent with the above, knockdown of SENP1 significantly decreased TNBC cell colony numbers (Fig. 2D and F). These results indicated that silencing of SENP1 could inhibit proliferation and colony formation of TNBC cells in vitro.

Depletion of SENPI inhibits the invasion, migration, and arrests TNBC cells in G1 phase of the cell cycle. The effects of SENP1 silencing on cell invasion and migration ability in TNBC cells were detected using Transwell and wound healing assay. As shown in Fig. 3A, silencing of SENP1 significantly reduced the invasion capacity of TNBC cells. Moreover, the results of wound healing assay showed suppressed migration capacity of si-SENP1 TNBC cells (Fig. 3B). Together, these results revealed the impaired invasion and migration ability of TNBC cells induced by SENP1 deficiency.

Additionally, to explore the mechanism of SENP1-mediated functions in cell cycle control, we monitored cell cycle synchronization of TNBC cells using FACS analysis. As shown in Fig. 3C, si-SENP1 in both MDA-MB-231 and MDA-MB-453 results in a higher percentage of cells in G1/G0 phase and a concomitant lower percentage of cells in G2/M stage and slight decrease in the percentage of cells in S phase. The results indicated that reduction of SENP1 would give rise to the inhibition of MDA-MB-231 and MDA-MB-453 cell proliferation mainly through modulating cell cycle progression.

SENP1 is required for the expression of $p 21, p 27$, and MMP9 in TNBC cells. Since that SENP-1 promotes metastasis through upregulating MMP9 in prostate and pancreatic cancer $(10,16)$, as well as that p21 and p27 were important G1 CDK inhibitors in breast cancer cell proliferation (18), the previous reports led us to investigate the effect of SENP1 in regulating the expression of p21, p27, and MMP9 in TNBC 
A

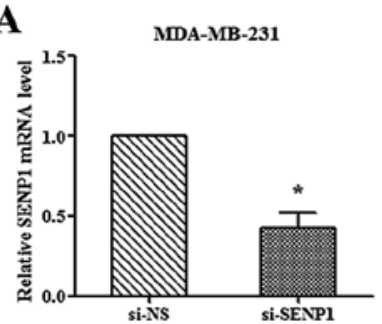

C

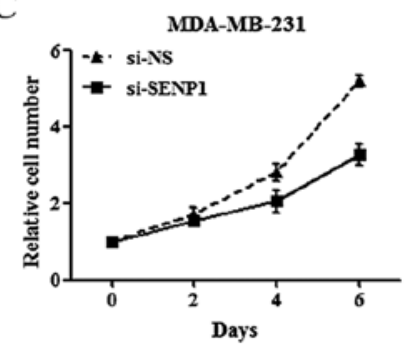

E

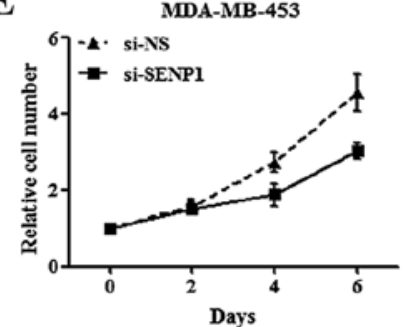

MDA-MB-453

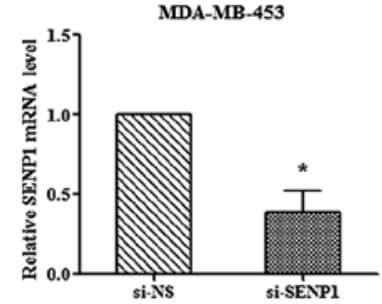

D

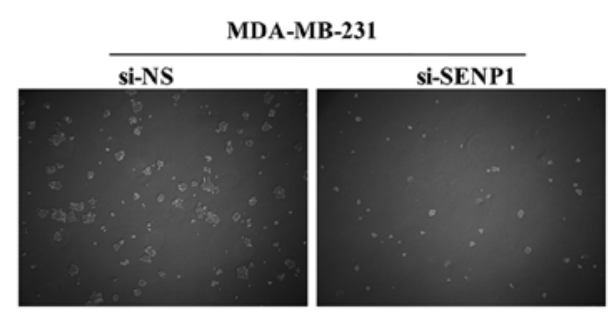

F

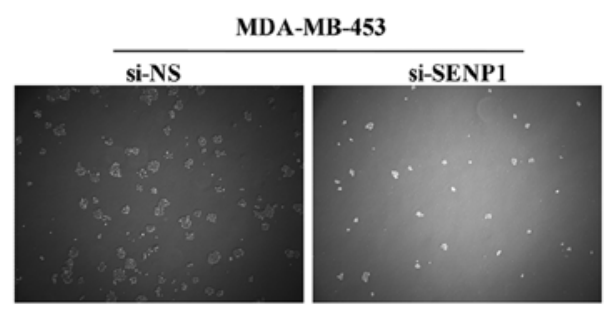

B

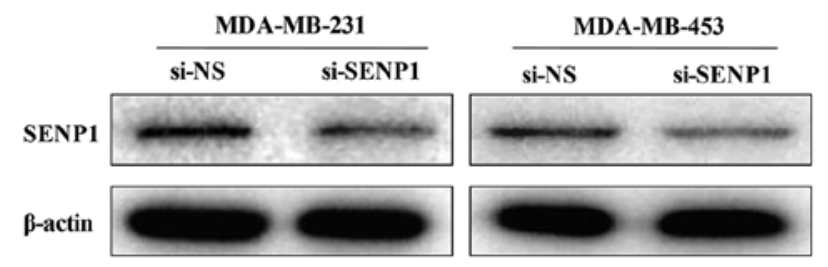

MDA-MB-231

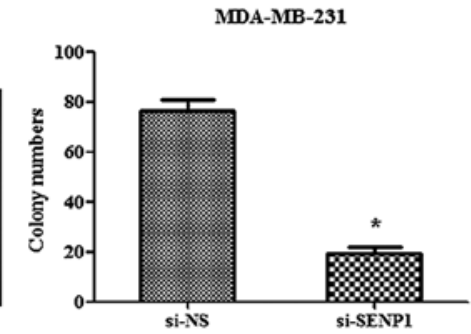

MDA-MB-453

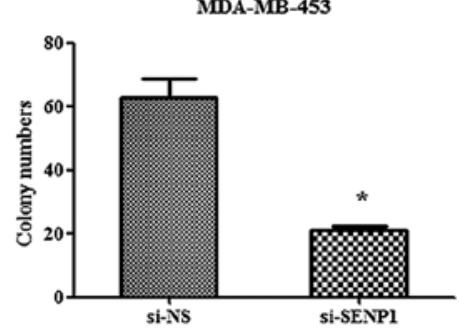

Figure 2. Knockdown of SENP1 inhibits the proliferation and colony formation of TNBC cells in vitro. (A) qRT-PCR analysis of SENP1 mRNA level in si-SENP1 TNBC cells. (B) Western blotting of SENP1 protein level in si-SENP1 TNBC cells. (C and E) Total cell numbers of (C) MDA-MB-231 and (E) MDA-MB-453 were counted. (D and F) Colony formations of si-SENP1 TNBC cell lines were determined 2 weeks after planting in soft agar. "P<0.05. SENP1, SUMO-specific protease 1; TNBC, triple-negative breast cancer.

cells. As shown in Fig. 3D and E, the mRNA and protein levels of p21 and p27 were significantly increased by SENP1 knockdown. However, the expression of MMP9 was decreased in the SENP1-depleting cells. These results indicated that SENP1 regulates cell proliferation of TNBC through controlling the expression of p21 and p27, and modulates cell invasion by altering the level of MMP9.

Silencing of SENP1 inhibits tumor growth and lung metastases in vivo. To explore the effect of SENP1 on TNBC tumor growth in vivo, cells transfected with si-NS and si-SENP1 were subcutaneously injected into nude mice, respectively. The volume of tumor was evaluated weekly from 1 to 8 weeks after transplantation. As shown in Fig. 4A and B, the tumor sizes in si-SENP1 group were significantly smaller than that in the control group. Pulmonary metastasis detection revealed that si-SENP1 cells had less lung metastases than si-NS control cells (Fig. 4C). Further, we performed IHC assay by using Ki67 antibody. We found that the expression level of Ki67 in SENP1-depleting tumor cells is lower than that in the control cells, which suggested that depletion of SENP1 would inhibit TNBC cell proliferation in vivo (Fig. 4D). Furthermore, as shown in Fig. 4E, the protein levels of p21, p27, and MMP9 were increased in tumor tissues from si-SENP1 TNBC cells in comparison with those from si-NS TNBC cells. These results elucidated the in vivo function of SENP1 in regulating TNBC cell proliferation and tumor formation.

\section{Discussion}

The SENPs play a pivotal role in maintaining ordered cellular physiology by modulating the balance between sumoylated and unsumoylated proteins. In mammalian cells, there are six identified SENP isoforms, namely SENP1, SENP2, SENP3, SENP5, SENP6, and SENP7, respectively. Emerging studies have demonstrated that various SENP isoforms were involved in the development of diverse diseases, such as pancreatic (16), colon (11), prostate $(19,20)$, bladder (21) and thyroid (22) cancer, as well as heart diseases (23). SENP1 is the first identified and most characterized SENP among the SENP family. Data from previous studies showed abnormal expression of SENP1 in several types of carcinomas. Wang et al reported that the expression of SENP1 is directly associated with CaP aggressiveness and recurrence. The absence of SENP1 restricted the tumor growth and metastasis in CaP cells (10). Moreover, SENP1 was increased in pancreatic cancer cells and knockdown of SENP1 inhibited the proliferation, migration, and invasion of pancreatic cancer cells (16). In this study, we found that SENP1 levels were markedly elevated in TNBC tissues. Through further in vitro and in vivo assays, we elucidated that knockdown of SENP1 modulated the proliferation, mobility, and cell cycle of TNBC cells.

Cell cycle was regulated by the activation of CDKs, which antagonized by the inhibitors, such as p21 and p27 $(24,25)$. The expression of p21 and p27 were reported to serve as 


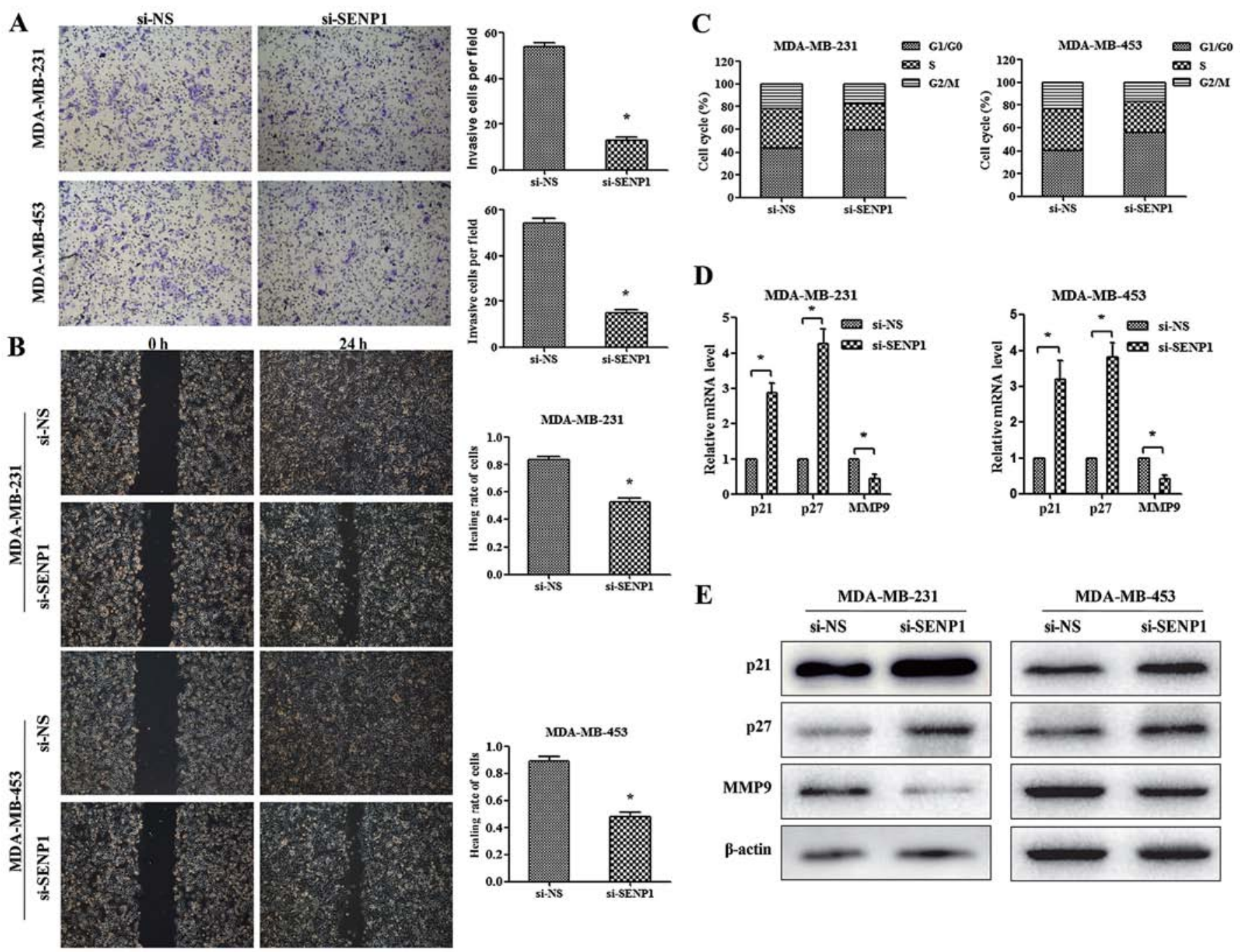

Figure 3. Silencing of SENP1 attenuated TNBC cell invasion, migration and arrested TNBC cells in G1 phase of the cell cycle. (A) Transwell assay was performed to measure the TNBC cell invasion transfected with si-SENP1 or si-NS. (B) The wound healing assay was applied to assess TNBC cell migration transfected with si-SENP1 or si-NS. The process of wound closure was photographed at 0 and $24 \mathrm{~h}$. (C) Flow cytometry analysis of the cell cycle distribution of si-SENP1 TNBC cell lines and the percentages of cells in G1/G0, S and G2/M phases were quantified. (D and E) Knockdown of SENP1 affected the expression of p21, p27 and MMP9. (D) qRT-PCR analysis of mRNA levels and (E) western blotting assay of protein levels of p21, p27, and MMP9 in TNBC cell lines transfected with si-SENP1 or si-NS. "P<0.05. SENP1, SUMO-specific protease 1; TNBC, triple-negative breast cancer.

A
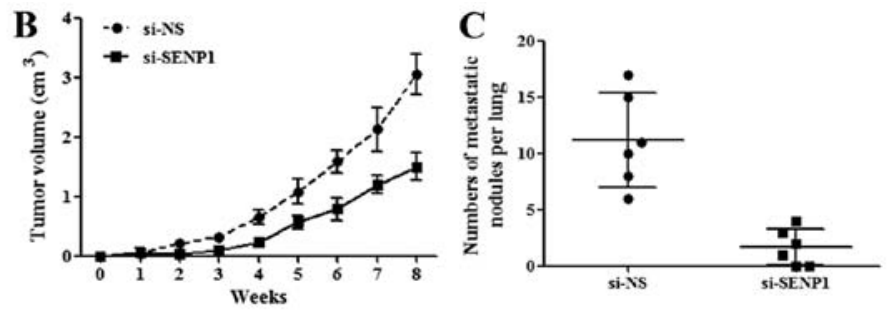

D

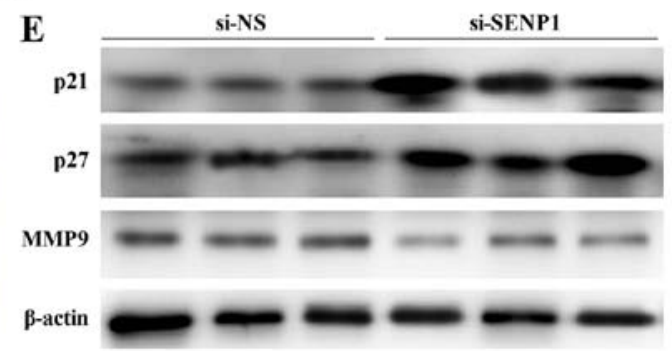

Figure 4. Knockdown of SENP1 reduced tumor growth in vivo. (A and B) Tumor volumes were evaluated in si-NS and si-SENP1 groups of injected mice 3 weeks after the si-SENP1 TNBC cells were injected into nude mice. (C) Number of metastatic lung foci detected in each group. (D) Immunohistochemical staining of Ki67, a marker for indicating proliferation (magnification, $\mathrm{x} 400$ ). (E) Western blotting of p21, p27, and MMP9 in these tumor samples with $\beta$-actin as a control. SENP1, SUMO-specific protease 1; TNBC, triple-negative breast cancer. 
the molecular basis for cell proliferation blockage in breast cancer cells $(18,26,27)$. For example, the signal transducer and activator of transcription 6 (STAT6) downregulates breast cancer cell proliferation by increasing the expression of $\mathrm{p} 21$ and p27 (18). To further verify the ability of SENP1 to mediate proliferation of TNBC cells, we investigated the expression levels of p21 and p27 in si-SENP1 TNBC cells. We found that silencing of SENP1 increased the expression of p21 and p27 in the two TNBC cell lines, MDA-MB-231 and MDA-MB-453, indicating that SENP1 regulates the proliferation of TNBC cells through repressing the expression of CDK inhibitors, p21, and p27. However, the mechanism of SENP1 in regulating the expression of p21 and p27 needs to be further investigated.

MMPs, the family of $\mathrm{Zn}$-dependent endopeptidases, are associated with the invasive properties of metastatic cancer cells $(28,29)$. It has been reported that MMP9 is overexpressed in breast cancer cells and is correlated with the invasion and metastasis of breast cancer $(30,31)$. Given that SENP1 affected the cancer invasion and metastasis, we further investigated whether the expression of MMP9 could be controlled by SENP1. Our data showed that knockdown of SENP1 resulted in MMP9 reduction both in MRNA and protein level. In addition, it has been demonstrated that SENP1 regulates the expression of MMP9 through HIF-1 $\alpha$ signaling in $\mathrm{CaP}$ cells (20). Thus, it could be an interesting point to confirm whether SENP1 regulates TNBC cell proliferation via HIF-1 $\alpha$ pathway.

In conclusion, we demonstrated that SENP1 was upregulated in TNBC tumor tissues when compared to adjacent normal and non-TNBC tumor tissues, respectively. Depletion of SENP1 attenuated TNBC cell proliferation, invasion, migration, and colony formation ability, and arrested TNBC cells in G1/G0 cell cycle progression in vitro, as well as the tumor size, tumor cell proliferation, and lung metastases in vivo. Furthermore, knockdown of SENP1 increased the expression of CKD inhibitors, p21 and p27, and decreased the expression of MMP9. Taken together, the current study elucidated a critical role of SENP1 in TNBC progression, which highlighted that SENP1 might be a potential therapeutic target for TNBC.

\section{Acknowledgements}

This study was supported by the Shanghai Municipal Science and Technology Commission Guidance Project, P.R. China (contract no. 14411966000).

\section{References}

1. Siegel R, Naishadham D and Jemal A: Cancer statistics, 2012. CA Cancer J Clin 62: 10-29, 2012.

2. Perou CM, Sørlie T, Eisen MB, van de Rijn M, Jeffrey SS, Rees CA, Pollack JR, Ross DT, Johnsen H, Akslen LA, et al: Molecular portraits of human breast tumours. Nature 406: 747-752, 2000.

3. Bauer KR, Brown M, Cress RD, Parise CA and Caggiano V: Descriptive analysis of estrogen receptor (ER)-negative, progesterone receptor (PR)-negative, and HER2-negative invasive breast cancer, the so-called triple-negative phenotype: A population-based study from the California Cancer Registry. Cancer 109: 1721-1728, 2007.

4. Elias AD: Triple-negative breast cancer: A short review. Am J Clin Oncol 33: 637-645, 2010.
5. Dent R, Trudeau M, Pritchard KI, Hanna WM, Kahn HK, Sawka CA, Lickley LA, Rawlinson E, Sun P and Narod SA: Triple-negative breast cancer: Clinical features and patterns of recurrence. Clin Cancer Res 13: 4429-4434, 2007.

6. Rakha EA, Elsheikh SE, Aleskandarany MA, Habashi HO, Green AR, Powe DG, El-Sayed ME, Benhasouna A, Brunet JS, Akslen LA, et al: Triple-negative breast cancer: Distinguishing between basal and nonbasal subtypes. Clin Cancer Res 15: 2302-2310, 2009.

7. Cheng J, Kang X, Zhang S and Yeh ET: SUMO-specific protease 1 is essential for stabilization of HIFlalpha during hypoxia. Cell 131: 584-595, 2007.

8. Li R, Wei J, Jiang C, Liu D, Deng L, Zhang K and Wang P: Akt SUMOylation regulates cell proliferation and tumorigenesis. Cancer Res 73: 5742-5753, 2013.

9. Li T, Huang S, Dong M, Gui Y and Wu D: Prognostic impact of SUMO-specific protease 1 (SENP1) in prostate cancer patients undergoing radical prostatectomy. Urol Oncol 31: 1539-1545, 2013.

10. Wang RT, Zhi XY, Zhang Y and Zhang J: Inhibition of SENP1 induces radiosensitization in lung cancer cells. Exp Ther Med 6: 1054-1058, 2013.

11. Xu Y, Li J,Zuo Y, Deng J, Wang LS and Chen GQ: SUMO-specific protease 1 regulates the in vitro and in vivo growth of colon cancer cells with the upregulated expression of CDK inhibitors. Cancer Lett 309: 78-84, 2011.

12. Burdelski C, Menan D, Tsourlakis MC, Kluth M, Hube-Magg C, Melling N, Minner S, Koop C, Graefen M, Heinzer H, et al: The prognostic value of SUMO1/Sentrin specific peptidase 1 (SENP1) in prostate cancer is limited to ERG-fusion positive tumors lacking PTEN deletion. BMC Cancer 15: 538, 2015.

13. Wang Q, Xia N, Li T, Xu Y, Zou Y, Zuo Y, Fan Q, Bawa-Khalfe T, Yeh ET and Cheng J: SUMO-specific protease 1 promotes prostate cancer progression and metastasis. Oncogene 32: 2493-2498, 2013.

14. Wang C, Tao W, Ni S, Chen Q, Zhao Z, Ma L, Fu Y and Jiao Z: Tumor-suppressive microRNA-145 induces growth arrest by targeting SENP1 in human prostate cancer cells. Cancer Sci 106: 375-382, 2015.

15. Yang H, Tang Y, Guo W, Du Y, Wang Y, Li P, Zang W, Yin X, Wang $\mathrm{H}$, Chu $\mathrm{H}$, et al: Up-regulation of microRNA-138 induce radiosensitization in lung cancer cells. Tumour Biol 35: 6557-6565, 2014.

16. Ma C, Wu B, Huang X, et al: SUMO-specific protease 1 regulates pancreatic cancer cell proliferation and invasion by targeting MMP-9. Tumour Biol 35: 12729-12735, 2014.

17. Huang BB, Gao QM, Liang W, Xiu B, Zhang WJ and Liang AB: Down-regulation of SENP1 expression increases apoptosis of Burkitt lymphoma cells. Asian Pac J Cancer Prev 13: 2045-2049, 2012.

18. Wei M, Liu B, Gu Q, Su L, Yu Y and Zhu Z: Stat6 cooperates with $\mathrm{Sp} 1$ in controlling breast cancer cell proliferation by modulating the expression of p21(Cip1/WAF1) and p27 (Kip1). Cell Oncol (Dordr) 36: 79-93, 2013.

19. Bawa-Khalfe T, Cheng J, Lin SH, Ittmann MM and Yeh ET: SENP1 induces prostatic intraepithelial neoplasia through multiple mechanisms. J Biol Chem 285: 25859-25866, 2010.

20. Kaikkonen S, Jääskeläinen T, Karvonen U, Rytinki MM, Makkonen H, Gioeli D, Paschal BM and JJ: SUMO-specific protease 1 (SENP1) reverses the hormone-augmented SUMOylation of androgen receptor and modulates gene responses in prostate cancer cells. Mol Endocrinol 23: 292-307, 2009.

21. Tan M, Gong H, Wang J, Tao L, Xu D, Bao E, Liu Z and Qiu J: SENP2 regulates MMP13 expression in a bladder cancer cell line through SUMOylation of TBL1/TBLR1. Sci Rep 5: 13996, 2015.

22. Jacques C, Baris O, Prunier-Mirebeau D, Savagner F, Rodien P, Rohmer V, Franc B, Guyetant S, Malthiery Y and Reynier P: Two-step differential expression analysis reveals a new set of genes involved in thyroid oncocytic tumors. J Clin Endocrinol Metab 90: 2314-2320, 2005.

23. Kim EY, Chen L, Ma Y, Yu W, Chang J, Moskowitz IP and Wang J: Enhanced desumoylation in murine hearts by overexpressed SENP2 leads to congenital heart defects and cardiac dysfunction. J Mol Cell Cardiol 52: 638-649, 2012.

24. Malumbres M and Barbacid M: Cell cycle, CDKs and cancer: A changing paradigm. Nat Rev Cancer 9: 153-166, 2009.

25. Shackelford RE, Kaufmann WK and Paules RS: Cell cycle control, checkpoint mechanisms, and genotoxic stress. Environ Health Perspect 107 (Suppl 1): 5-24, 1999. 
26. Groshong SD, Owen GI, Grimison B, Schauer IE, Todd MC Langan TA, Sclafani RA, Lange CA and Horwitz KB: Biphasic regulation of breast cancer cell growth by progesterone: Role of the cyclin-dependent kinase inhibitors, p21 and p27(Kip1). Mol Endocrinol 11: 1593-1607, 1997.

27. Wang X, Gao P, Long M, Lin F, Wei JX, Ren JH, Yan L, He T, Han Y and Zhang HZ: Essential role of cell cycle regulatory genes p21 and p27 expression in inhibition of breast cancer cells by arsenic trioxide. Med Oncol 28: 1225-1254, 2011.

28. Akter H, Park M, Kwon OS, Song EJ, Park WS and Kang MJ: Activation of matrix metalloproteinase-9 (MMP-9) by neurotensin promotes cell invasion and migration through ERK pathway in gastric cancer. Tumour Biol 36: 6053-6062, 2015.
29. Kessenbrock K, Plaks V and Werb Z: Matrix metalloproteinases: Regulators of the tumor microenvironment. Cell 141: 52-67, 2010.

30. Shi M, Cao M, Song J, Liu Q, Li H, Meng F, Pan Z, Bai J and Zheng J: PinX1 inhibits the invasion and metastasis of human breast cancer via suppressing NF- $\kappa \mathrm{B} / \mathrm{MMP}-9$ signaling pathway. Mol Cancer 14: 66, 2015.

31. Sun YS, Zhao Z and Zhu HP: Hispolon inhibits TPA-induced invasion by reducing MMP-9 expression through the NF- $\kappa B$ signaling pathway in MDA-MB-231 human breast cancer cells. Oncol Lett 10: 536-542, 2015. 